\title{
Modelado de la dispersión de material particulado en la ciudad de Los Ángeles (Chile) a partir de las estufas a leña en el período de invierno usando AERMOD
}

\section{Modelling of particulate matter dispersion in Los Angeles city (Chile) from wood stoves in winter period using AERMOD}

Fecha de entrega: 10 de junio 2016 Fecha de aceptación: 7 de septiembre 2016

\section{Adrián Silva ${ }^{1}$ y Gustavo Godoy ${ }^{2}$}

${ }^{1}$ Departamento de Ciencias y Tecnología Vegetal, Escuela de Ciencias y Tecnologías, Universidad de Concepción, Juan Antonio Coloma 0210, Los Ángeles, Chile, asilvaf@udec.cl

${ }^{2}$ Departamento de Ciencias Geodésicas y Geomática, Escuela de Ciencias y Tecnologías, Universidad de Concepción, Juan Antonio Coloma 0210, Los Ángeles, Chile, gustavogodoy@udec.cl

La contaminación atmosférica es un desafio importante para la autoridad ambiental en Chile principalmente en las ciudades del sur, entre ellas la ciudad de Los Ángeles. Se utilizó el modelo AERMOD para determinar puntos de máximo impacto, factores de emisión óptimos e influencia de barrios en la ciudad de Los Ángeles, para material particulado MP2.5 y MP10, debido al uso de estufas residenciales a leña. El modelo utiliza datos de las fuentes urbanas, datos meteorológicos para años 2013 y 2014, y topografía digital. Los puntos de máximo impacto para concentración de 24 horas se ubican en las coordenadas WGS 84 UTM Husol8 $733045 \mathrm{mE}$ y $5849051 \mathrm{mS}$ (2013) y $733345 \mathrm{mE}$ y 5849451 $m S$ (2014) correspondientes al barrio Hurtado. Los factores de emisión óptimos obtenidos fueron $0.69 \mathrm{~g}_{\text {MP2.5 }} / \mathrm{kg}_{\text {leña }}$ y de 2.1 $g_{\text {MPIO }} / \mathrm{kg}_{\text {leña }}$ niveles para los cuales equipos a pellets o gas son aptos. Los barrios más influentes sobre los puntos de mayor impacto son Pueblo Nuevo y Centro con una participación de $59 \%$ y $13.5 \%$ respectivamente, por lo que la calidad del aire en el barrio Hurtado tiene una importante influencia de barrios adyacentes.

Palabras clave: modelo AERMOD, MP2.5, MP10, estufas residenciales a leña, calidad del aire
Air pollution is a major challenge for the environmental authorities in Chile, mainly in southern cities, including Los Angeles city. AERMOD model was used to determine points of maximum impact, optimal emission factors and influence of neighbourhoods in Los Angeles city, for particulate matter PM2.5 and PM10, because of the use of residential wood stoves. Model uses data from urban sources, meteorological data for year 2013 and 2014, and digital topography. Points of maximum impact for 24-hour concentration are located at WGS 84 UTM coordinates Zone18 $733045 \mathrm{mE}$ and $5849051 \mathrm{mS}$ (2013) and $733345 \mathrm{mE}$ and $5849451 \mathrm{mS}$ (2014) for Hurtado neighbourhood. Optimal emission factors obtained were $0.69 \mathrm{~g}_{\text {PM2.5 }} / \mathrm{kg}_{\text {wood }}$ and $2.1 \mathrm{~g}_{\text {PMIO }} / \mathrm{kg}_{\text {wood }}$, levels for which pellets or gas equipments are suitable. Neighbourhoods that have greater influence on points of maximum impact are Pueblo Nuevo and Central with a share of $59 \%$ and $13.5 \%$ respectively, so that air quality in the neighbourhood Hurtado has an important influence of adjacent neighbourhoods.

Keywords: AERMOD model, PM2.5, PM10, residential wood stoves, air quality

\section{Introducción}

La contaminación atmosférica es el principal desafío para la autoridad ambiental en Chile ya que ocasiona al menos 4 mil muertes prematuras a nivel nacional. Actualmente, se mide la contaminación en más de 25 ciudades, cuyas concentraciones de contaminación crecen hacia el sur, generándose la necesidad de establecer e implementar 14 Planes de Descontaminación Atmosférica (PDA) entre los años 2014 y 2018 (MMA, 2014).
El año 2015 fue oficialmente declarada la comuna de Los Ángeles, en la Región del Bío Bío en el sur de Chile, como zona saturada a causa de los elevados niveles de contaminación atmosférica que se han presentado durante varios años en los meses del invierno, por emisiones de los materiales particulados respirables MP2.5 y MP10 provenientes de la combustión de estufas a leña (MMA, 2015). Esto resulta preocupante ya que los estudios han puesto de manifiesto la variabilidad espacial sustancial 
en las relaciones entre la exposición a largo plazo a la contaminación del aire exterior y la salud (Singh et al., 2014). Experiencias internacionales indican que en muchas ciudades las concentraciones en masa de MP10 siguen superando los factores de calidad del aire (Cyrys et al., 2014) y que la combustión de madera es una de las fuentes locales dominantes de MP2.5, junto con fuentes como el tráfico de vehículos y emisiones (Gidhagen et al., 2015; Ginzburg et al., 2015).

En el caso de la ciudad de Los Ángeles, debido a los altos niveles de contaminación, el Ministerio del Medio Ambiente MMA tiene la responsabilidad de elaborar y ejecutar un PDA para la comuna, que considere la definición e implementación de medidas y acciones específicas con la finalidad de recuperar la calidad ambiental del territorio y la calidad de vida de los habitantes, puesto que existe una amplia literatura que relaciona los niveles de MP10 y MP2.5 con efectos negativos en la salud (Qui y Peng, 2015; CONAMA, 2007). Algunas de las medidas y acciones específicas que podrán proponerse en el PDA son el aumento del porcentaje de áreas verdes, un programa de recambio voluntario de sistemas de calefacción a leña por equipos de calefacción más eficientes con menores emisiones de partículas y el mejoramiento de la aislación térmica de viviendas.

Previamente a la implementación de cualquier tipo de política ambiental en un área urbana es necesario realizar un diagnóstico de la situación ambiental, que en el caso particular de la calidad del aire, consiste en determinar cuantitativamente mediante mediciones o estimaciones de las concentraciones de contaminantes en el aire (Mazzeo y Venegas, 2004). El uso de modelos de dispersión de emisiones atmosféricas para estimaciones de concentraciones de contaminantes en el aire, está ampliamente difundido internacionalmente y se han aplicado principalmente para determinar los efectos, sobre territorios poblados, de las emisiones provenientes de fuentes emisoras de tipo puntual ubicadas en zonas industriales cercanas. No obstante, también pueden encontrarse trabajos sobre el uso de modelos de dispersión de emisiones atmosféricas primarias por fuentes urbanas superficiales y puntuales, con el objetivo de entregar antecedentes para toma de decisiones políticas en materia de regulación y protección ambiental. Por ejemplo, Bealey et al. (2007), utilizando un modelo de dispersión de calidad del aire (dispersión y deposición), cuantificaron para dos ciudades del Reino Unido, la magnitud potencial de reducción de la concentración ambiental de MP10 alcanzable a través de la plantación de árboles urbanos. Las salidas del modelo se incorporaron en un Sistema de Información Ambiental para la toma de decisiones sobre planeación del uso de la tierra en planes de desarrollo y planeación estratégica y para el control del desarrollo urbano. También, Moudi et al. (2011), realizaron un estudio de caso de la sensibilidad de la concentración de contaminantes a variables meteorológicas y a parámetros de uso de la tierra sobre la ciudad de Douala en Camerún, usando un modelo de dispersión atmosférica. Los resultados ayudan a elegir con precisión las áreas indicadas para la implantación de instalaciones industriales, para gestionar la evaluación de riesgos a la exposición de contaminantes en la ciudad y para direccionar recomendaciones a los responsables de las políticas. En Latinoamérica, puede mencionarse el trabajo de Mazzeo y Venegas (2004), quienes presentaron el diseño de un sistema de vigilancia para un contaminante primario en el aire en la ciudad de Buenos Aires, que incluyó la aplicación de un modelo de dispersión atmosférica, con el fin de determinar las áreas urbanas y la población afectada, posibilitando que las autoridades cuenten con una metodología de detección del deterioro de la calidad del aire urbana y puedan planificar las acciones necesarias para disminuir sus consecuencias. En Chile, la utilización de un modelo de dispersión atmosférica para material particulado puede encontrarse en el trabajo realizado por Silva y Arcos (2011) en la ciudad de Constitución. Se determinó que la tasa de emisión de equipos calefactores a leña debe ser inferior a $7.5 \mathrm{~g} / \mathrm{h}$ MP10 para no superar la norma vigente para MP10 en promedios de 24 horas en ningún punto de la ciudad, y que tasas mayores de emisión en rangos de 10 a 22.5 y de 25 a $40 \mathrm{~g} / \mathrm{h}$ de MP10 tendrían como consecuencia la superación de la norma en la ciudad en 1 y 2 puntos respectivamente. También, Carrasco et al. (2011), utilizando un modelo de dispersión de calidad del aire, evaluaron en la ciudad de Rancagua el beneficio ambiental de la calefacción distrital con base en la biomasa frente a los actuales calefactores a leña, como alternativa para la reducción de emisiones de material particulado fino MP2.5. 
En el trabajo de modelación de dispersión que se presenta a continuación, se utiliza un modelo de dispersión gaussiano el cual permite simular y describir el material particulado atmosférico en la comuna de Los Ángeles. Este tipo de modelo, a nivel comunal, forma parte de los principios necesarios para una evaluación integral de inventarios de emisiones y se utilizan para verificar que las emisiones sean consistentes con el impacto registrado por un monitoreo (USACH, 2014). Específicamente, el modelo de dispersión gaussiano AERMOD, es un modelo reglamentario que fue especialmente diseñado para apoyar los programas de modelado de regulación de Environmental Protection Agency EPA de EEUU (Thé et al., 2014). Se han realizado diversas publicaciones con el objetivo de comparar este modelo con bases de datos reales y con otros modelos, obteniéndose, con pocas excepciones, rendimientos superiores en comparación con otros modelos testeados (Perry et al., 2005). Por ejemplo, Hadlocon et al. (2015) realizaron un estudio similar sobre la dispersión de partículas de una instalación avícola, y se llegó a la conclusión de que el modelo AERMOD puede predecir eficazmente las concentraciones de material particulado atmosférico.

Como resultado de este trabajo, se entrega información que es importante que sea considerada para la toma de decisiones técnicas y políticas respecto de las medidas y acciones específicas que se deban implementar para conseguir una adecuada gestión ambiental del territorio saturado con MP2.5 y MP10 debido a la combustión residencial de leña en período invernal. Esto permite conocer los puntos de máximo impacto ambiental dentro de la ciudad. Además se pueden determinar los máximos factores de emisión a los cuales deben operar los equipos a combustión a leña para que los niveles de MP2.5 y MP10 no superen los valores máximos como concentración diaria. También se identifica la influencia que tiene cada barrio de la ciudad sobre la concentración de MP2.5 y MP10 en los puntos de mayor impacto, teniendo en consideración para los parámetros respectivos, los límites establecidos para las concentraciones de 24 horas de los Decretos Supremos $\mathrm{N}^{\mathrm{o}} 12 / 12$ y No20/13 del Ministerio del Medio Ambiente de Chile (MMA, 2012, 2013).

\section{Desarrollo metodológico}

El modelo AERMOD EPA calcula la distribución de los contaminantes en una pluma de manera horizontal y vertical siguiendo un comportamiento de tipo normal denominado distribución de Gauss (US EPA, 2004), incorporando algoritmos de dispersión para considerar las diferencias de cotas del terreno en el dominio (terreno complejo). Uno de los insumos básicos para AERMOD es el archivo de configuración de flujos que contiene las opciones seleccionadas de modelado, así como la ubicación de origen y de los parámetros de datos, ubicaciones de los receptores, especificaciones de archivos de datos meteorológicos, y opciones de salida. Para aplicaciones que implican efectos de terreno elevado, se necesitan los datos del receptor y del terreno (US EPA, 2004).

\section{Tasa de emisión en zona urbana (datos de las fuentes emisoras)}

Dominio de la modelación:

El área o dominio de modelación considerada en este estudio corresponde a la que se muestra en Figura 1, cuyas posiciones de los vértices fueron determinadas mediante coordenadas UTM Huso 18 WGS 84. Desde su origen en el punto SW, el dominio considera $10 \mathrm{~km}$ en sentido Este y $16.5 \mathrm{~km}$ en sentido Norte, cubriendo una superficie de $165 \mathrm{~km}^{2}$, en la que se incluye la zona urbana y los sectores rurales adyacentes a la ciudad de Los Ángeles.

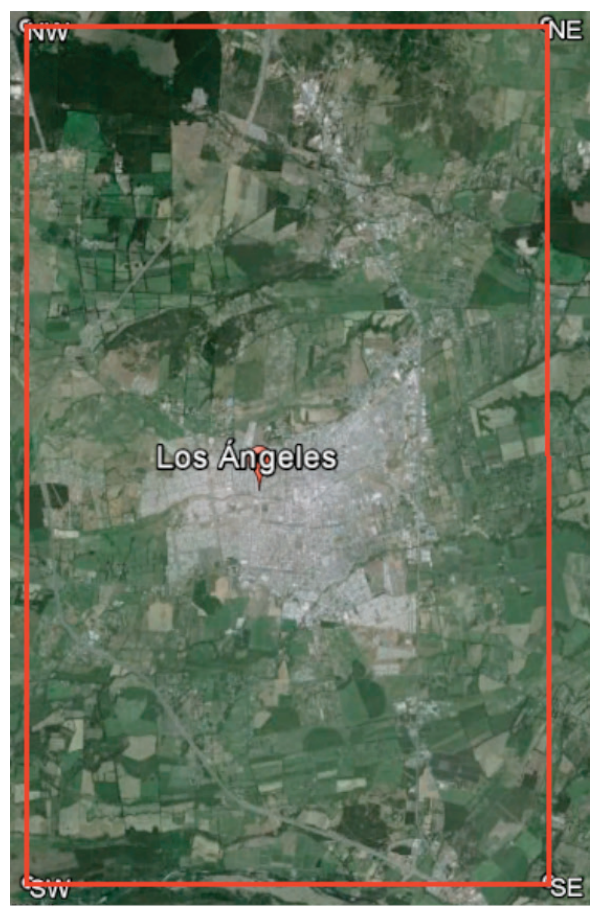

Figura 1: Ilustración del área de dominio utilizada en la modelación 


\section{Emisiones areales:}

Las emisiones areales dependen de la superficie y de la densidad de personas utilizando equipos de combustión a leña en los diferentes barrios de la ciudad, lo que está relacionado con la condición socioeconómica de sus habitantes. La ciudad de Los Ángeles cuenta con 17 barrios cuyas superficies se muestran en la Figura 2.

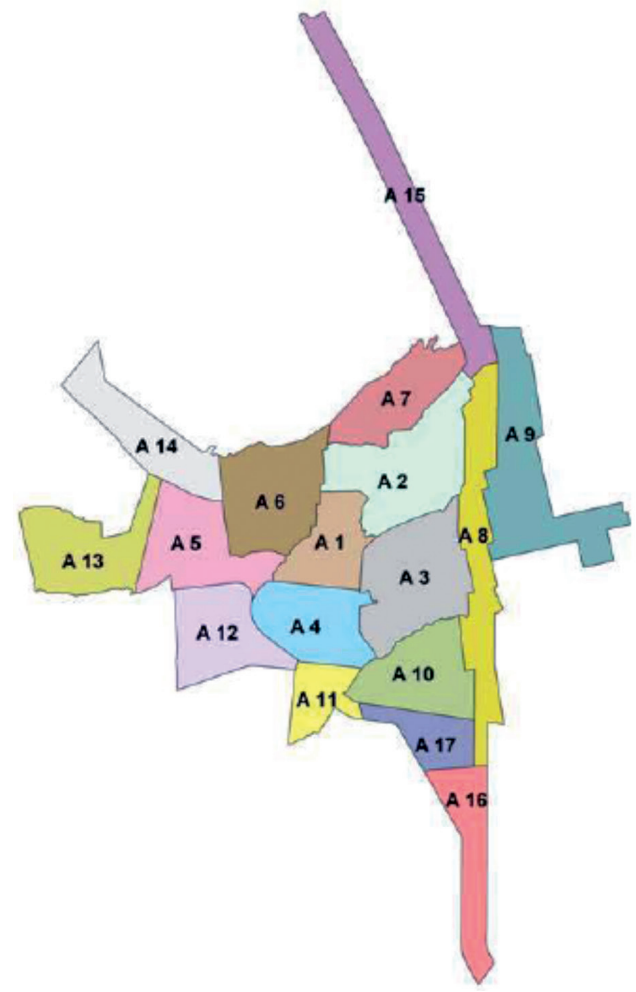

\begin{tabular}{|c|l|r|}
\hline ID & Nombre & Superficie (há) \\
\hline A 1 & Barrio Centro & 153,73 \\
\hline A 2 & Barrio Alemania & 298,74 \\
\hline A 3 & Barrio Pueblo Nuevo & 203,21 \\
\hline A 4 & Barrio Hurtado & 232,54 \\
\hline A 5 & Barrio Orompello & 262,39 \\
\hline A 6 & Barrio Sor Vicenta & 314,22 \\
\hline A 7 & Barrio Las Industrias & 276,31 \\
\hline A 8 & Barrio Oriente & 347,28 \\
\hline A 9 & Barrio Francisco Encina & 212,22 \\
\hline A 10 & Barrio Sur & 99,00 \\
\hline A 11 & Barrio El Retiro & 206,87 \\
\hline A 12 & Barrio Extensión Poniente & 235,93 \\
\hline A 13 & Barrio Extensión Maria Dolores & 205,49 \\
\hline A 14 & Barrio Extensión El Avellano & 188,20 \\
\hline A 15 & Barrio Extensión Longitudinal Norte & 259,48 \\
\hline A 16 & Barrio Extensión Longitudinal Sur & 169,08 \\
\hline A 17 & Barrio Extensión Tolpán & 108,38 \\
\hline SUPERFICIE TOTAL & $3.773,07$ \\
\hline
\end{tabular}

Figura 2: Distribución de barrios y superficies en la zona urbana de la ciudad de Los Ángeles. (Municipalidad de Los Ángeles, 2005)

Para incorporar estas coberturas al modelo fue utilizado un archivo electrónico de formato vectorial de almacenamiento digital de polígonos con los barrios de la comuna. De los polígonos se obtuvo los vértices, y luego éstos se simplificaron por el criterio de la diferencia entre la pendiente de aristas contiguas (Figura 3). Para la simplificación de los polígonos se aplicó un filtro para la eliminación de vértices, cuyo criterio específico utilizado fue la diferencia entre la pendiente de dos aristas contiguas. Si la diferencia es despreciable, entonces se elimina el punto entre las dos pendientes. El proceso es:

$i_{1}=\frac{Y_{2}-Y_{1}}{X_{2}-X_{1}} ; \quad i_{2}=\frac{Y_{3}-Y_{2}}{X_{2}-X_{2}}$

$\Delta i=i_{1}-i_{2}$, si $\Delta i<0.05$, se elimina el punto.

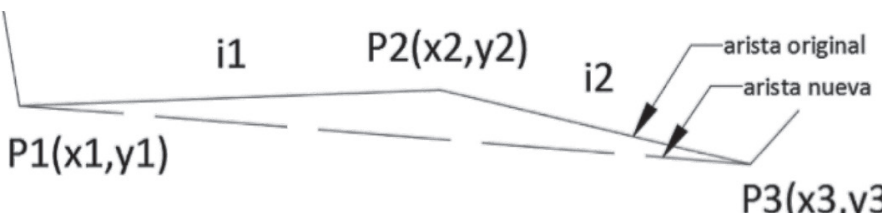

Figura 3: Esquema de simplificación de aristas de un polígono

\section{Fuentes emisoras:}

Se consideran fuentes emisoras aquellas viviendas que sean del tipo casas (excluyendo otro tipo de viviendas como departamentos y piezas) y que se encuentren en la zona urbana de la ciudad. El número de casas en la zona urbana se determina utilizando datos de reportes estadísticos oficiales (Casen 2009, Censo 2002 y Censo 2012) reportados por BCN $(2012)$ e INE $(2009,2012)$, en donde se consideran las variables total de viviendas, viviendas del tipo casa y el factor demográfico urbano - rural. De esta forma, se obtiene que en la comuna de Los Ángeles existen 40464 casas en la zona urbana. Para la modelación, las casas se encuentran distribuidas espacialmente por barrios de acuerdo a la forma que establece la Municipalidad de Los Ángeles (2005). Por otra parte, estudios realizados en la ciudad de Temuco, que pueden ser extrapolables a la ciudad de Los Ángeles por su cercanía, similares condiciones climáticas y socioeconómicas, señalan que el $100 \%$ de los hogares utilizan preferentemente leña como sistema de calefacción (Lobos, 2001). Considerando lo anterior, puede considerarse que en la ciudad de Los Ángeles existen 40464 fuentes emisoras (equipos) a combustión a leña. 
Factor de usos equipos de combustión a leña:

La leña en período invernal se utiliza en promedio 19 horas al día, siendo su distribución según estrato socioeconómico alto, medio y bajo de 20, 21 y 14 horas respectivamente (Lobos, 2001), con lo que se obtienen factores de uso de equipos de $0.83,0.88$ y 0.58 horas de operación por cada hora del día, respectivamente.

\section{Tasa de emisión}

La tasa de emisión corresponde al producto entre la tasa de actividad o de quemado de leña y el factor de emisión del equipo calefactor (US EPA, 1995).

\section{Tasa de quemado de leña:}

La tasa de quemado, considerando las diversas variables para la operación de un equipo de calefacción a leña, puede establecerse entre 1.7 a $3.6 \mathrm{~kg}$ de leña por hora de operación (CONAMA, 2002). Para este estudio, se utiliza una tasa de quemado con valores en un rango entre $1.5 \mathrm{a}$ $3.5 \mathrm{~kg}$ de leña por hora de operación, distribuyendo este valor según el nivel socioeconómico promedio de los habitantes de los barrios de la ciudad.

\section{Factores de emisión en equipos de combustión:}

El factor de emisión corresponde a un valor representativo que intenta relacionar la cantidad de un contaminante emitido a la atmósfera con una actividad asociada con la liberación de dicho contaminante. Se expresa normalmente como el peso de contaminante dividido por una unidad de peso, volumen, distancia o la duración de la actividad emisora del contaminante (US EPA, 1995). Datos de USACH (2014) establecen Factores de Emisión para MP10 de $15.3 \mathrm{~g} / \mathrm{kg}$ para salamandras y estufas simples y de $9.8 \mathrm{~g} / \mathrm{kg}$ para estufas de doble entrada, los cuales junto a los datos corregidos de los estudios de CONAMA (2007) que indican que en la región del Bío Bío existe un $28 \%$ de salamandras, un $43.6 \%$ de equipos de combustión simple y un $25.7 \%$ de equipos de combustión doble, puede establecerse un Factor de Emisión promedio para el estudio de $14 \mathrm{~g}_{\mathrm{MP} 10}$ por $\mathrm{kg}$ de leña consumida. Por otra parte, considerando la relación peso/peso de 95 a $97 \%$ entre MP2.5 y MP10 informadas en los datos del USACH (2014) y de CONAMA (2009), se obtiene un Factor de Emisión de $13 \mathrm{~g}_{\mathrm{MP} 2,5}$ por $\mathrm{kg}$ de leña consumida para las fuentes areales residenciales.
De acuerdo a US EPA (2004) la expresión de la concentración de un parámetro es directamente proporcional a la tasa de emisión de la fuente, por lo tanto, a partir de los valores de máxima concentración es posible determinar los máximos factores de emisión de equipos de combustión para los materiales particulados respirables MP2.5 y MP10.

\section{Información meteorológica}

La información meteorológica considerada en el estudio, corresponde a la registrada por la Estación Meteorológica 21 de Mayo de la ciudad de Los Ángeles, perteneciente al Sistema de Información Nacional de Calidad del Aire SINCAdel Ministerio de Medio Ambiente, con coordenadas UTM Huso $18733331 \mathrm{mE} 5849585$ mS y complementada con datos de las estaciones Las Viñas, Los Ángeles y Tierras Nobles, cercanas a la ciudad y pertenecientes a la Red Agroclima FDF-INIA-DMC. La serie temporal de las condiciones atmosféricas considera datos meteorológicos cada 60 minutos durante los días 21 de junio al 21 de septiembre, para los años 2013 y 2014. Las variables meteorologías utilizadas son: dirección y magnitud del viento, temperatura del aire, presión atmosférica, humedad relativa, radiación solar, precipitación y cobertura de nubes. Para este último parámetro, dado que no existen registros para la ciudad de Los Ángeles, se consideró cobertura total, consistente con la alta cobertura de nubes existente en el período invernal. Los datos meteorológicos se validaron y se pre-procesaron mediante el software AERMET View 9.0, incorporando la información resultante en el software AERMOD View 9.0 de dispersión atmosférica.

\section{Escenario topográfico}

La dispersión de contaminantes es influenciada a escala local por características de la superficie (US EPA, 2004), por lo cual el uso del suelo es un factor que incide en la dispersión y el arrastre de elementos contaminantes a través del aire. En efecto, dado que el resultado de esta modelación se utilizó como referencia para la calidad del aire de la población, se otorga mayor relevancia a la dispersión en el área urbana. Se consideran para el período invernal, valores para albedo, radio de bowen y rugosidad de superficie para zona urbana. Para la modelación se utilizó topografía digital Shuttle Radar Topography Mission SRTM, de 3 segundos de arco, para la ciudad de Los Ángeles. Ver Figura 4. 


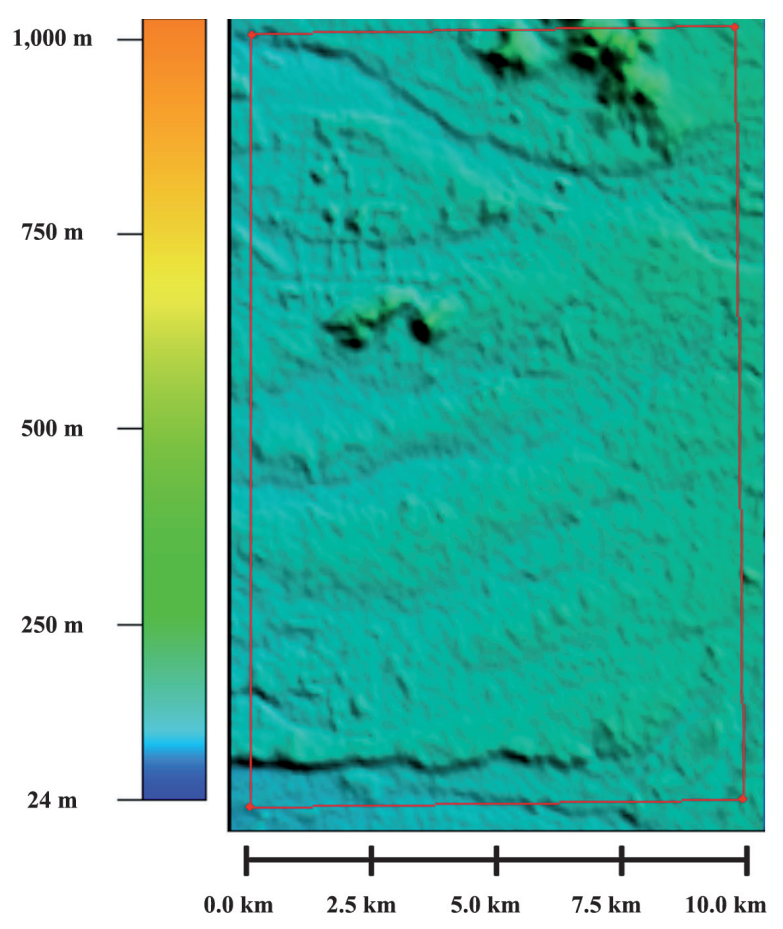

Figura 4: Topografía SRTM de Los Ángeles

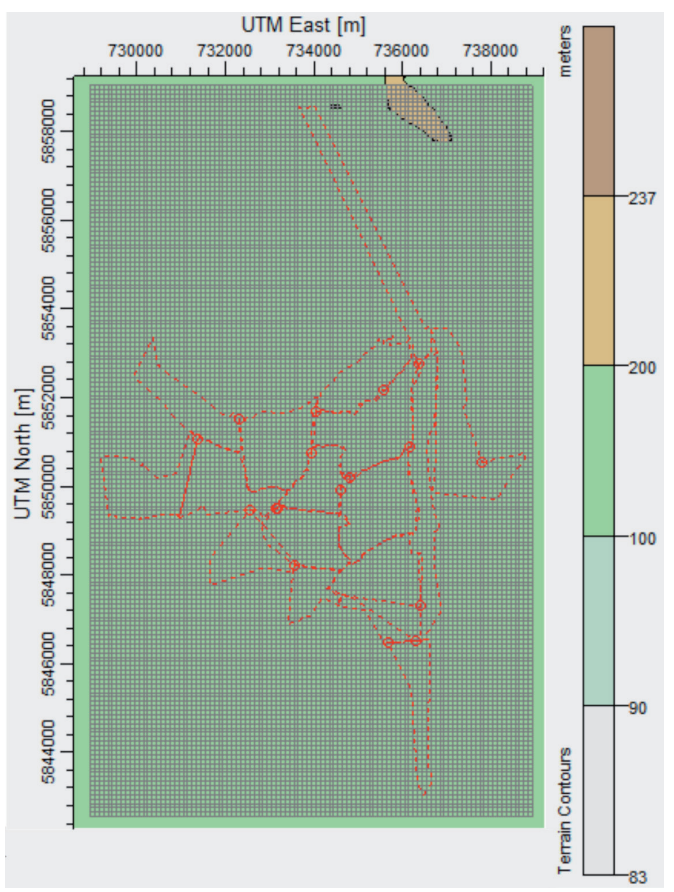

Figura 5: Topografía procesada en AERMAP

La imagen digital de topografía de la Figura 4, es procesada mediante el software AERMAP. Sobre esta imagen procesada y para efectos de la implementación del sistema de modelación con el modelo AERMOD, se consideró una grilla de 10 x $16.5 \mathrm{~km}$ con cuadrículas de 100 x $100 \mathrm{~m}$ cada una (Figura 5).

\section{Resultados}

Puntos de máximo impacto para MP2.5y MP10 para los años 2013 y 2014

Detalles de la dispersión de la concentración de MP2.5 y MP10 para 24 horas y para el período invernal se muestran en las Figuras 6, 7, 8 y 9. Estas muestran un comportamiento general de mayores concentraciones en los barrios interiores de la ciudad para las concentraciones de 24 horas, con superación de los valores máximos normados por la legislación chilena de $50 \mu \mathrm{g} / \mathrm{m}^{3}$ para MP2.5 en todos los barrios y de $150 \mu \mathrm{g} / \mathrm{m}^{3}$ para MP10 en los barrios centrales (Figuras 6 y 8), con una leve tendencia a la cobertura de las mayores concentraciones hacia barrios de oriente para la modelación del periodo invernal (Figuras 7 y 9). Se observa además que, para el año 2013, para ambos parámetros, el punto de mayor impacto para la concentración de 24 horas se ubica en la coordenada $733045 \mathrm{mE}$ y $5849051 \mathrm{mS}$ y para el período de invierno en la coordenada $733345 \mathrm{mE}$ y 5849451 mS. Para el año 2014, para ambos parámetros, el punto de mayor impacto para la concentración de 24 horas y para el período invernal se ubica en la coordenada 733345 $\mathrm{mE}$ y $5849451 \mathrm{mS}$. Los puntos de mayor impacto se ubican ambos en el barrio Hurtado (A4) en las intersecciones de las calles Daniel de La Vega con Eduardo Barrios (año 2013) y Ricardo Vicuña con Camilo Henríquez (año 2014). Los máximos valores de concentración de 24 horas fueron alcanzados en el mes de julio.

\section{Factores de emisión óptimos de materiales particulados respirables MP2.5 y MP10 para equipos de combustión a leña}

Para el promedio de 24 horas, las concentraciones máximas de MP2.5 y MP10 son respectivamente para el año 2013 de 935 y $985 \mu \mathrm{g} / \mathrm{m}^{3}$. Ambos datos para el año 2014 son 953 y $1003 \mu \mathrm{g} / \mathrm{m}^{3}$. De acuerdo a esto, promediando los respectivos parámetros y considerando las concentraciones máximas de 24 horas de acuerdo a las normativas de calidad, se obtiene que los factores de emisión óptimos promedio para equipos de combustión a leña, para que en conjunto sus tasas de emisión no superen los valores de concentración normados $\left(50 \mu \mathrm{g} / \mathrm{m}^{3}\right.$ para MP2.5 y $150 \mu \mathrm{g} /$ $\mathrm{m}^{3}$ para MP10), son de $0.69 \mathrm{~g}_{\mathrm{MP} 2,5} / \mathrm{kg}_{\text {leña }}$ y de $2.1 \mathrm{~g}_{\mathrm{MP10}} / \mathrm{kg}_{\text {leña }}$. 


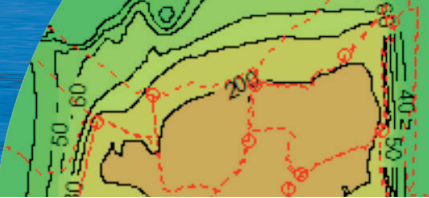

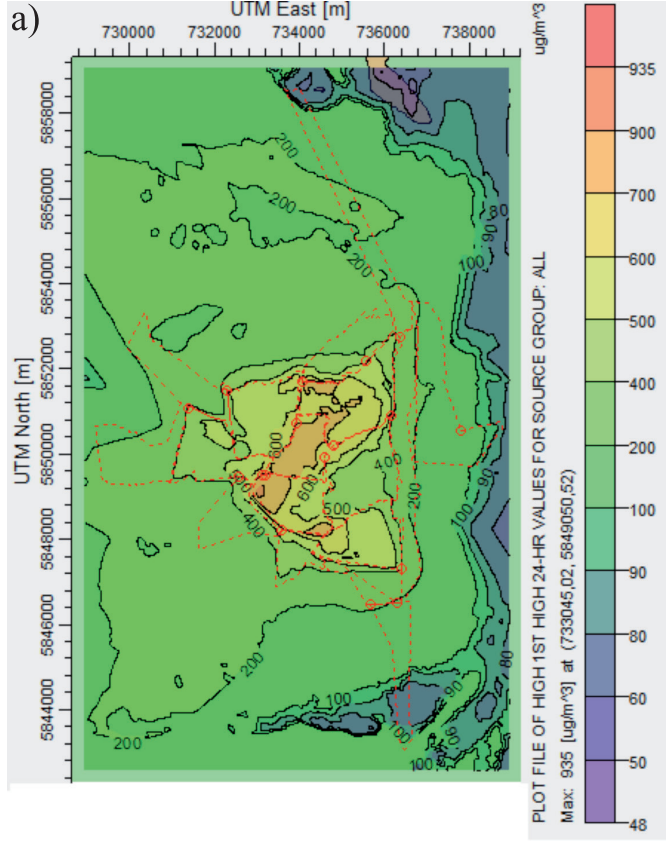

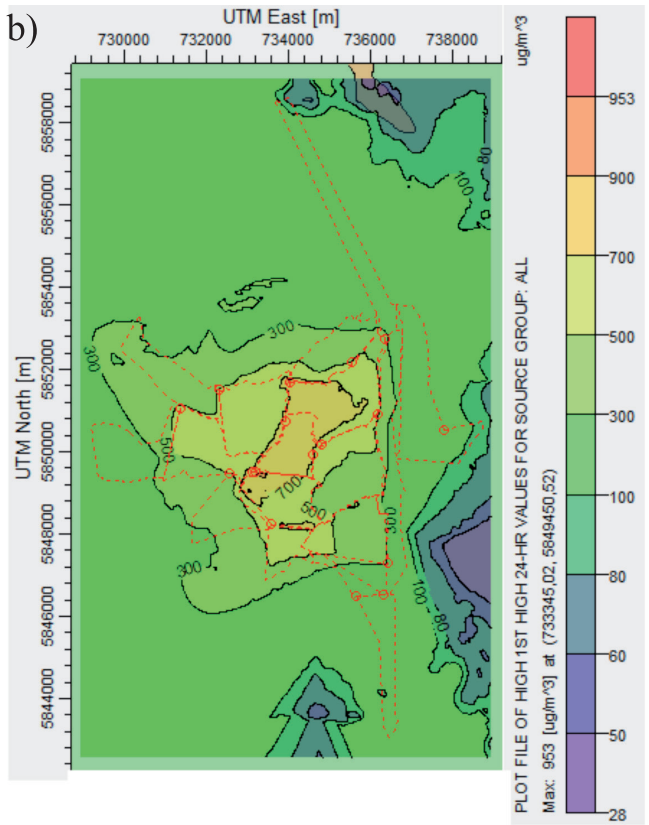

Figura 6: MP2.5 de 24 horas, a) año 2013 y b) año 2014

Influencias de barrios en los puntos de mayor impacto La influencia de los diferentes barrios de la ciudad en los puntos de mayor impacto para promedios de 24 horas se muestra en la Figura 10. Se observa que los barrios que tienen mayor influencia sobre la concentración de material particulado respirable son A3 y A1 con una participación promedio para los años 2013-2014 de 59\% y $13.5 \%$ respectivamente, considerando que sus respectivas
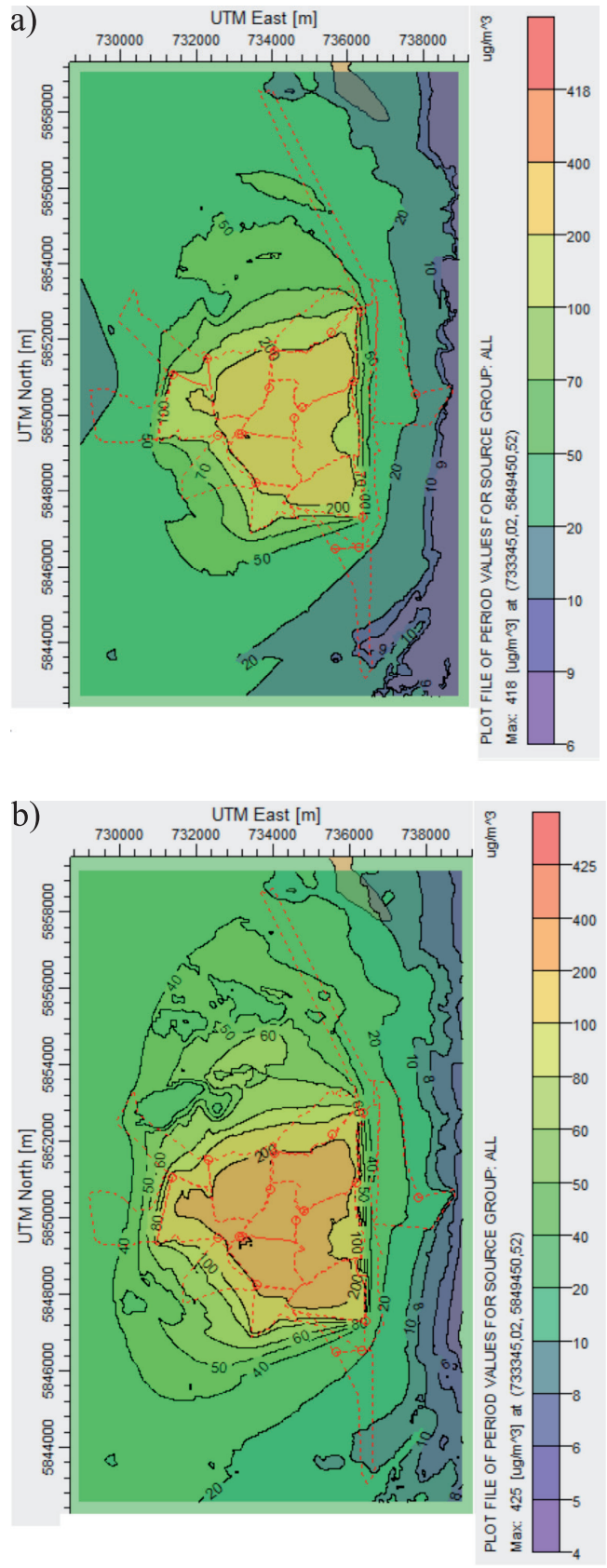

Figura 7: MP2.5 del periodo invernal, a) año 2013 y b) año 2014

participaciones en la tasa de emisión global son de $22 \%$ y 14\% (Figura 11). Para los demás barrios, los aportes en la concentración en los puntos de mayor impacto son mucho menores en relación a sus tasas de emisión, donde inclusive el barrio A4, que es aquel en donde se encuentran los puntos de mayor impacto, su influencia en la concentración es de $4 \%$, sin embargo su participación en las tasa de emisión global es de $8 \%$. 

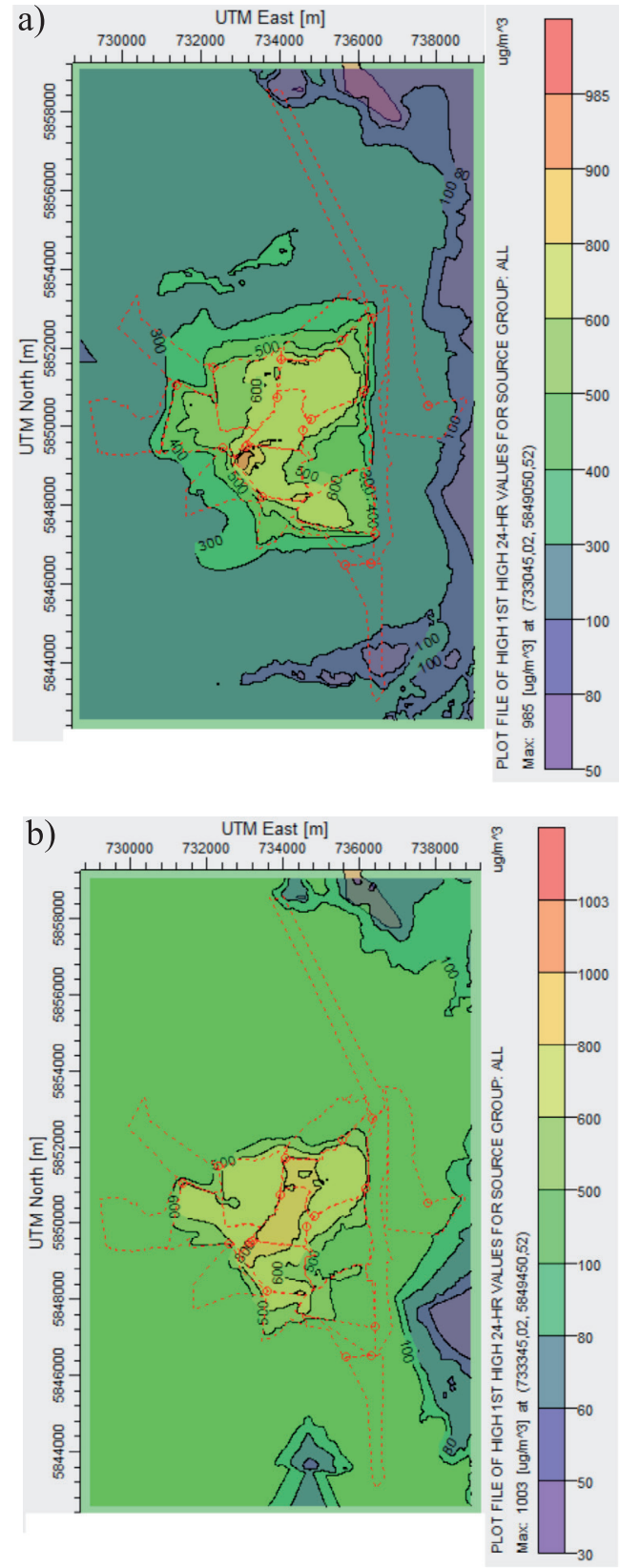

Figura 8: MP10 de 24 horas, a) año 2013 y b) año 2014

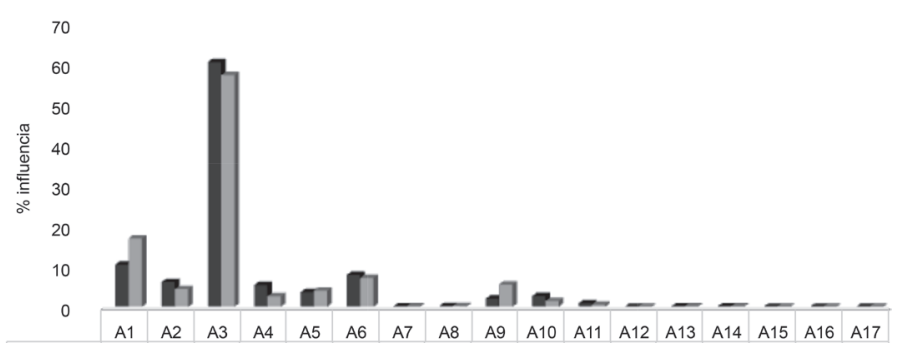

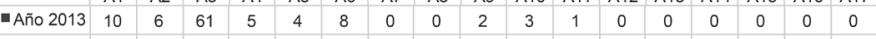
\begin{tabular}{|l|l|l|l|l|l|l|l|l|l|l|l|l|l|l|l|l|l|} 
- Año 2014 & 17 & 4 & 57 & 3 & 4 & 7 & 0 & 0 & 5 & 1 & 0 & 0 & 0 & 0 & 0 & 0 & 0 \\
\hline
\end{tabular} Barrios

Figura 10: Gráfico de la influencia de barrios en la concentración de materiales particulados respirables para promedios de 24 horas en punto de mayor impacto, años 2013 y 2014
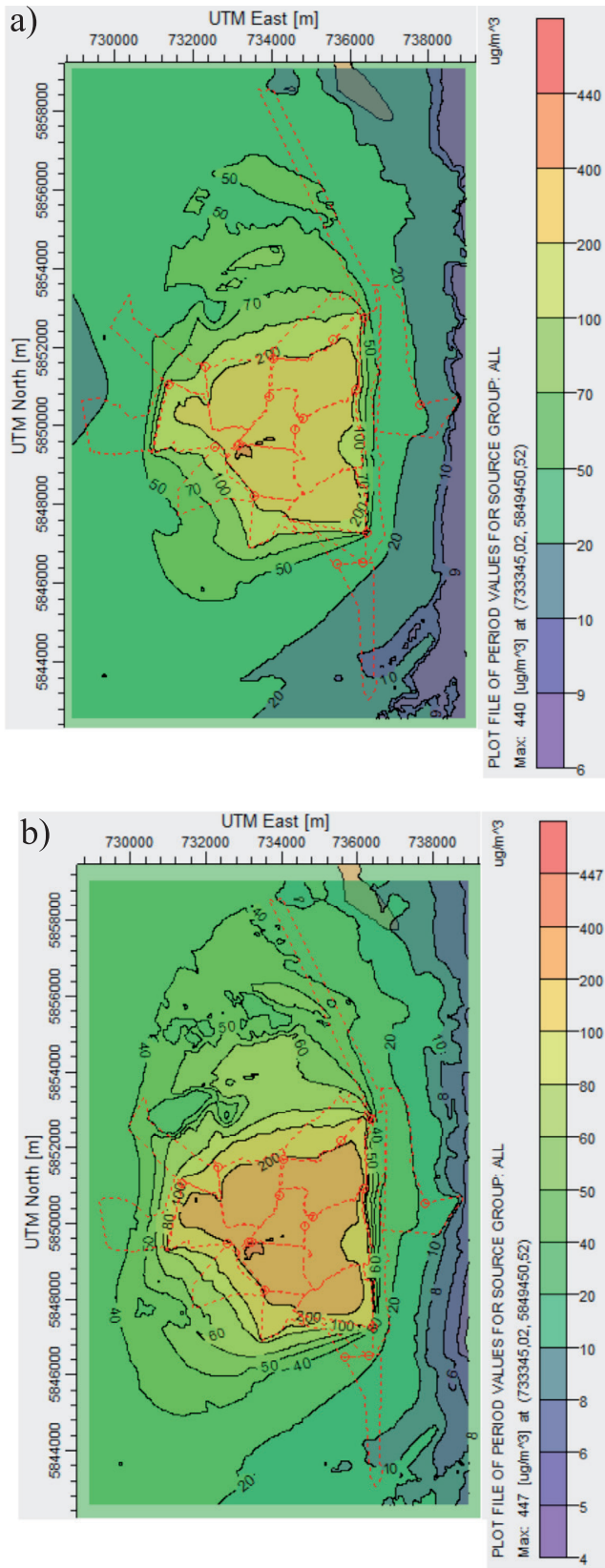

Figura 9: MP10 del periodo invernal, a) año 2013 y b) año 2014

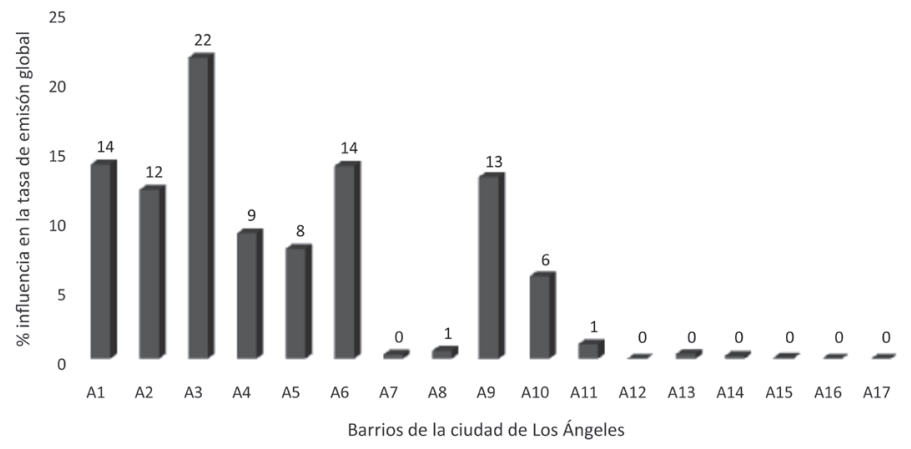

Figura 11: Gráfico de la participación de barrios en la tasa de emisión global de materiales particulados respirables 


\section{Discusiones}

Los resultados de este estudio sugieren que se requiere validar los datos de la modelación con datos reales medidos en la ciudad, sin embargo, las dos estaciones de monitoreo de la calidad del aire actualmente en operaciones, no resultan ser suficientes para cubrir representativamente toda el área geográfica modelada. Los datos de concentraciones resultantes, pueden ser inferiores en situaciones de menor estabilidad atmosférica por menor cobertura nubosa. También, existe la posibilidad de la utilización no simultánea de todos los equipos a leña, lo cual puede implicar una reducción en la concentración de MP2.5 y MP10 resultante de la simulación.

Por otro lado, debe tenerse en consideración que la concentración de MP2.5 y MP10 resultante, también se ve influenciada por el nivel basal de ambos parámetros que genera una concentración base adicional no considerada en la simulación. Además las fuentes residenciales aumentan permanentemente y existen fuentes emisoras que no han sido consideradas, como por ejemplo las fuentes industriales y fuentes móviles, lo que puede implicar un aumento de la concentración de MP2.5 y MP10. También, es importante señalar que los niveles de emisiones pueden ser más altos si se considera el uso del tiraje por parte de los usuarios. Encuestas en varias ciudades del centro-sur de Chile han mostrado que el $99 \%$ de los hogares utiliza el tiraje cerrado o semi-cerrado para que la leña dure más. Esta práctica de ahogar la combustión hace que los equipos a leña emitan más materiales particulados (Schueftan y González, 2015).

Otra situación que debe tenerse en consideración, tiene relación con que la contaminación de la combustión residencial de leña es una preocupación importante en áreas con topografía de valle, en donde inversiones térmicas durante la noche limitan la dispersión de contaminantes procedentes de fuentes de origen terrestre (Allen et al., 2011). Dado que en la noche la estabilidad atmosférica varía de neutra a moderadamente estable (De Nevers, 1998), es muy probable que la concentración de materiales particulados sea mayor en ese período del día.

En cuanto a los factores de emisión óptimos para el caso de MP10, el único tipo de calefactor a biomasa que puede satisfacer factores de emisión iguales o bajo
$2.1 \mathrm{~g}_{\mathrm{MP} 10} / \mathrm{kg}_{\text {leña }}$ son las estufas a pellets, según datos de factores de emisión informados por USACH (2014) o bien equipos a gas, ya que presentan menores emisiones frente a combustibles líquidos y biomasa (GLP Chile, 2011). En cuanto al MP2.5, de acuerdo a lo informado por SINIA (2011), son los equipos a gas aquellos que tienen factores de emisión capaces de satisfacer tales exigencias. Otras alternativas de calefacción que es necesario investigar y evaluar su implementación, corresponden a los sistemas de calefacción distrital con uso de biomasa como combustible y las bombas de calor en base a energía eléctrica solar.

\section{Conclusiones}

Los puntos de mayor impacto se encuentran en el barrio Hurtado (A4) en una ubicación hacia el interior de la ciudad de Los Ángeles, con concentraciones modeladas que superan altamente los valores normados de 24 horas para las normas primarias de calidad ambiental asociadas a los materiales respirables MP2.5 y MP10. Teniendo presente lo anterior, resultaría beneficioso considerar en el PDA, aumentar el porcentaje de áreas verdes preferentemente en los barrios del interior de la ciudad para facilitar procesos de deposición de materiales particulados atmosféricos sobre la cobertura vegetal.

Los factores máximos de emisiones promedio que deben considerar los equipos de combustión a leña para que en conjunto sus tasas de emisión no superen los niveles de calidad del aire normados para MP2.5 y MP10, son muy bajos, para lo cual es necesario considerar equipos altamente eficientes que utilicen combustibles tales como pellets o gas. Por lo cual, al incluir en el PDA un programa de recambio de sistemas de calefacción en la ciudad, este debe considerar equipos con iguales o menores emisiones de materiales particulados respirables que los equipos a pellets o gas.

Los puntos de mayor impacto se trasladaron espacialmente a un barrio del interior de la ciudad (A4) que tiene una baja influencia en las tasas de emisión de materiales particulados atmosféricos a nivel global y no se presentaron en los barrios con más altas tasas de emisión (A3 y A1). Sin embargo, estos últimos resultaron ser altamente influyentes en las altas concentraciones de materiales particulados respirables en los puntos de mayor impacto. Este resultado puede considerarse en el PDA para orientar las inversiones 
en aislación térmica de viviendas hacia aquellos barrios que tienen mayor influencia en la deficiente calidad del aire de la ciudad.

Los valores de materiales particulados son en general altos para toda la ciudad en concentraciones de 24 horas, motivo por el cual, la mejora de la aislación térmica con el objetivo de evitar pérdidas de calor y evitar infiltraciones de MP2.5 y MP10 al interior de los hogares, debe ser una medida de aplicación general. Los puntos de mayor impacto pueden ser tomados como lugares de referencia para evaluar, por parte del Ministerio del Medio Ambiente, la instalación de nuevas estaciones de monitoreo de la calidad del aire en la ciudad de Los Ángeles.

\section{Agradecimientos}

Los autores agradecen particularmente a Lakes Environmental Software, por facilitar la adquisición de la licencia educacional del software AERMOD View, a la Red Agroclima FDF-INIA-DMC, por facilitar datos meteorológicos de las estaciones meteorológicas Los Ángeles, La Viñas y Tierras Nobles, y a la Secretaría Comunal de Planificación de la Ilustre Municipalidad de Los Ángeles, por facilitar archivos electrónicos de formato vectorial de almacenamiento digital de los barrios de la ciudad de Los Ángeles.

\section{Referencias}

Allen, G.A., Miller, P.J., Rector, L.J., Brauer, M. and Su, J.G. (2011). Characterization of valley winter woodsmoke concentrations in Northern NY using highly time-resolved measurements. Aerosol and Air Quality Resarch 11(5), 519-530

Bealey, W., McDonald, A., Nemitz, E., Donovan, R., Dragosits, U., Duffy, T. and Fowler, D. (2007). Estimating the reduction of urban PM10 concentrations by trees within an environmental information system for planners. Journal of Environmental Management 85(1), 44-58

BCN (2012). Reportes Estadísticos Comunales 2012 - Comuna de Los Ángeles. Biblioteca del Congreso Nacional de Chile

Carrasco, R., Jiménez del Rio, J. y Mardones, C. (2016). Análisis costo-beneficio de la calefacción distrital en la Zona Central de Chile. Revista Internacional de Contaminación Ambiental 32 (1), 35-45

CONAMA (2009). Inventario de emisiones atmosféricas para las ciudades de Chillán y Los Ángeles. Comisión Nacional del Medio Ambiente, Región del Bío Bío, estudio desarrollado por la Universidad Católica de Temuco

CONAMA (2007). Análisis técnico-económico de la aplicación de una norma de emisión para artefactos de uso residencial que combustionan con leña y otros combustibles de biomasa. Comisión Nacional del Medio Ambiente, estudio desarrollado por Ambiente Consultores.

CONAMA (2002). Priorización de medidas de reducción de emisiones por uso residencial de leña para la gestión de la calidad del aire en Temuco y Padre Las Casas. Comisión Nacional del Medio Ambiente, Región de la Araucanía, estudio desarrollado por Universidad de Concepción

Cyrys, J., Peters, A., Soentgen, J. and Wichmann, H.E. (2014). Low emission zones reduce PM10 mass concentrations and diesel soot in German cities. Journal of the Air \& Waste Management Association 64(4), 481-487

De Nevers, N. (1998). Ingeniería de control de la contaminación del aire. Mac Graw-Hill Interamericana, México

Ginzburg, H., Liu, X., Baker, M., Shreeve, R., Jayanty, R. K. M., Campbell, D. and Zielinska, B. (2015). Monitoring study of the near-road PM2.5 concentrations in Maryland. Journal of the Air \& Waste Management Association 65(9), 1062-1071

Gidhagen, L., Bennet, C., Segersson, D. and Omstedt, G. (2015). Exposure modeling of traffic and wood combustion emissions in Northern Sweden. In Environmental Software Systems. Infrastructures, Services and Applications. Springer International Publishing, 242-251

GLP Chile (2011). Evaluación de impacto atmosférico de sistemas de calefacción domiciliaria. Estudio desarrollado por el Centro Nacional del Medio Ambiente de la Universidad de Chile, Informe $\mathrm{N}^{\circ} 1$

Hadlocon, L.S., Zhao, L.Y., Bohrer, G., Kenny, W., Garrity, S.R., Wang, J., Wyslouzil, B. and Upadhyay, J. (2015). Modeling of particulate matter dispersion from a poultry facility using AERMOD. Journal of the Air \& Waste Management Association 65(2), 206-217

INE (2009). Población: Publicaciones y Estudios - Región del Biobío. Instituto Nacional de Estadísticas de Chile

INE (2012). Resultados XVIII Censo de Población 2012. 
Características demográficas, sociales, culturales y económicas de la población. Instituto Nacional de Estadísticas de Chile, Tomo I

Lobos, M. (2001). Estudio preliminar, sobre producción, comercialización y consumo de leña en la ciudad de Temuco. WWF/CODEFF. Documento $\mathrm{N}^{\circ} 3$, Serie de Publicaciones WWF, Programa Ecoregión Valdiviana

Mazzeo, N. y Venegas, L. (2004). Diseño de un sistema de vigilancia de NOx en la ciudad de Buenos Aires. Congreso de Saneamiento y Medio Ambiente - Desafios ambientales y del saneamiento en el siglo XXI, AIDIS, Buenos Aires, Argentina

MMA (2012). Decreto 12 establece norma primaria de calidad ambiental para material particulado fino respirable $\mathrm{MP}_{2,5}$. Ministerio del Medio Ambiente, Biblioteca del Congreso Nacional de Chile

MMA (2013). Decreto 20 establece norma de calidad primaria para material particulado respirable $\mathrm{MP}_{10}$, en especial de los valores que definen situaciones de emergencia y deroga decreto $\mathrm{n}^{\circ} 59$ de 1998 del Ministerio Secretaría General de la Presidencia. Ministerio del Medio Ambiente, Biblioteca del Congreso Nacional de Chile

MMA (2015). Decreto 11 declara zona saturada por material particulado fino respirables $\mathrm{MP}_{2.5} \mathrm{y}$ por material particulado respirables $\mathrm{MP}_{10}$, ambas como concentración diaria, a la comuna de Los Ángeles. Ministerio del Medio Ambiente, Biblioteca del Congreso Nacional de Chile

MMA (2014). Planes de Descontaminación Atmosférica PDA Estrategia 2014 - 2018. Ministerio del Medio Ambiente de Chile Municipalidad de Los Ángeles (2005). Plan regulador comunal de Los Ángeles - Memoria Explicativa.

Moudi, P., Appolinaire, D. and Mkankam, F. (2011). Case study of pollutants concentration sensitivity to meteorological fields and land use parameters over Douala (Cameroon) using AERMOD dispersion model. Atmosphere 2(4), 715-741

Perry, S.G., Cimorelli, A.J., Paine, R.J., Brode, R.W., Weil,
J.C., Venkatram, A., Wilson, R.B., Lee, R.F. and Peters, W.D. (2005). AERMOD: A dispersion model for industrial source applications. Part II: Model performance against 17 field study databases. Journal of Applied Meteorology 44(5), 694-708

Qiu, Z. and Peng, X. (2015). Investigating the impact of urban grade-separation on pedestrian PM2.5 exposure. Clean Technologies and Environmental Policy 17(7), 1917-1927

Schueftan. A. and González. A. (2015). Proposals to enhance thermal efficiency programs and air pollution control in SouthCentral Chile. Energy Policy 79, 48-57

Singh, V., Sokhi, R. S. and Kukkonen, J. (2014). PM2.5 concentrations in London for 2008-A modeling analysis of contributions from road traffic. Journal of the Air \& Waste Management Association 64(5), 509-518

SINIA (2011). Guía metodológica inventario de emisiones atmosféricas: M11 Metodología SINCA 2011. Sistema Nacional de Información Ambiental, Ambiosis S.A., Santiago

Silva, A. y Arcos, D. (2011). Aplicación del programa AERMOD para modelar dispersión de PM10 emitido por equipos de calefacción a leña en la ciudad de Constitución. Obras y Proyectos 9, 4-10

Thé, J.L., Thé, C.L. and Johnson, M.A. (2014). AERMOD view user guide. Lakes Environmental Software, Waterloo, Canada

USACH (2014). Actualización y sistematización del inventario de emisiones de contaminantes atmosféricos en la Región Metropolitana. Departamento de Física, Universidad de Santiago de Chile

US EPA (1995). AP 42 - Compilation of air pollutant emission factors: Stationary Point and Area Sources. Environmental Protection Agency, Volumen 1, Fifth Edition.

US EPA (2004). AERMOD, description of model formulation. Environmental Protection Agency, Staff Report, EPA454/R-03-004, North Carolina, USA 